\section{Case Reports in Oncology}

\title{
A Case of Primary Hepatic Neuroendocrine Tumor and Literature Review
}

\author{
Pak Chiu Wong $^{\text {a }}$ Wong Hoi She ${ }^{a}$ Ui Soon Khoo ${ }^{b}$ Tan To Cheung ${ }^{a}$ \\ aDepartment of Surgery, Queen Mary Hospital, The University of Hong Kong, Hong Kong \\ SAR, China; 'bepartment of Pathology, Queen Mary Hospital, The University of Hong Kong, \\ Hong Kong SAR, China
}

\section{Keywords}

Primary hepatic neuroendocrine tumor · Liver neoplasm · Carcinoid tumor

\begin{abstract}
We report an unusual presentation of primary hepatic neuroendocrine tumor which was initially misdiagnosed as intrahepatic cholangiocarcinoma. The diagnosis was only revealed after a major liver resection by histopathology. With adjuvant lanreotide injection, the patient survived for more than 16 months after the operation without tumor recurrence. Diagnosis of this rare tumor has been a major challenge and we emphasize the importance of a preoperative diagnosis. Surgical resection remains the mainstay for curative treatment, while peptide receptor radionuclide therapy is an emerging treatment option which has provided promising results.

\section{Background}

Neuroendocrine tumors (NETs) of the liver represent a rare entity. Within this group of uncommon diseases, primary hepatic NET (PHNET) only represented $0.3 \%$ of all NETs [1]. Less than 150 cases were reported up to 2017, and information on the disease demographics, diagnostic, and management strategies were uncertain. Herein, we report a case of PHNET in a patient with an uncommon presentation. 


\section{Case Reports in Oncology}

\begin{tabular}{l|l}
\hline Case Rep Oncol 2021;14:90-97 \\
\hline DOI: 10.1159/000510935 & $\begin{array}{l}\text { ○ 2021 The Author(s). Published by S. Karger AG, Basel } \\
\text { www.karger.com/cro }\end{array}$ \\
\hline
\end{tabular}

Wong et al.: Diagnostic Difficulties of a Rare Tumor
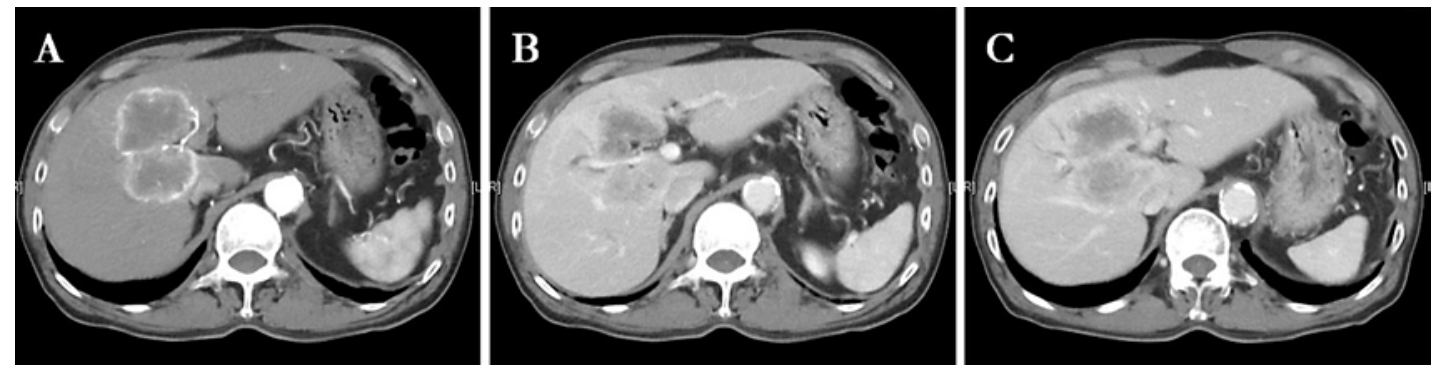

Fig. 1. A $5.7 \times 7.1 \times 6.4-\mathrm{cm}$ ill-defined heterogeneous mass with mild peripheral arterial enhancement $(\mathbf{A})$ followed by gradual centripetal filling (B) was noted in segments IV, V, and VIII. There was invasion of the left and right hepatic ducts and their confluence, and the proximal common bile duct, resulting in mildly dilated left and right intrahepatic ducts (C).

\section{Case Presentation}

A 69-year-old man presented to us for an incidental finding of a liver mass during an admission for pneumonia. He was a hepatitis B carrier with history of hypertension and impaired fasting glucose. He was asymptomatic with no abdominal pain, diarrhea, flushing, jaundice, or weight loss. Initial blood tests showed normal liver function and tumor markers including alphafetoprotein, carcinoembryonic antigen, and carcinoma antigen 19.9. Contrast-enhanced computed tomography (CT) of the abdomen showed a $5.7 \times 7.1 \times 6.4-\mathrm{cm}$ ill-defined heterogeneous mass occupying segment IV, V, and VIII with mild peripheral arterial enhancement followed by gradual centripetal filling (Fig. 1A, B). The tumor was found invading into the confluence of right and left hepatic ducts and the proximal common bile duct (Fig. 1C). The anterior segmental branch of the right portal vein was encased and compressed by the mass, while the left and main portal veins were patent. Based on the imaging findings, the patient was treated as intrahepatic mass-forming cholangiocarcinoma. Hepatocellular carcinoma (HCC) was another possible differential diagnosis given the background of underlying chronic hepatitis $\mathrm{B}$ infection. ${ }^{18} \mathrm{~F}$-fluorodeoxyglucose (FDG) positron emission tomography (PET) showed a hypermetabolic liver tumor with a maximum standardized uptake value (SUVmax) of 9.6 (Fig. 2). Several mildly hypermetabolic porta hepatis and aortocaval lymph nodes with SUVmax values $<1.7$ were noted, which were inconclusive of nodal metastases. No other primary tumor or distant metastatic lesions were identified. The indocyanine green clearance test was $9.46 \%$ at $15 \mathrm{~min}$. The left lateral section volume was $282 \mathrm{~mL}$, which corresponded to 27 and $26 \%$ of the estimated standard liver volume by the Urata formula [2] and HKU formula [3], respectively.

Open right trisectionectomy with left hepaticojejunostomy was performed. Postoperative recovery was uneventful. Pathological examination of the specimen found an $8 \times 6 \times$ 5 -cm solitary firm tan-white mass with no satellite nodules. There was presence of lymphovascular permeation and the liver parenchymal resection margin was involved (Fig. 3). Further microscopic analysis revealed tumor cells with oval nuclei containing dispersed chromatin, prominent nucleoli, distinct nuclear and cytoplasmic membranes forming tubules or nests with eosinophilic cytoplasm separated by fine fibrous bands (Fig. 4, 5). Fibrodesmoplasia and necrosis was found toward the central area. Mitotic figures were 8-10 per 10 highpower fields. Immunohistochemical stainings showed the tumor was diffusely positive for chromogranin (Fig. 6), and focally positive for synaptophysin and neuron-specific enolase. The tumor was negative for CD56, CK20, and Hepar1 (Fig. 7). The MIB-1 proliferation index was $3-5 \%$. The overall diagnosis was compatible with a moderately differentiated NET. According to the 2013 North American Neuroendocrine Tumor Society (NANETS) Consensus 

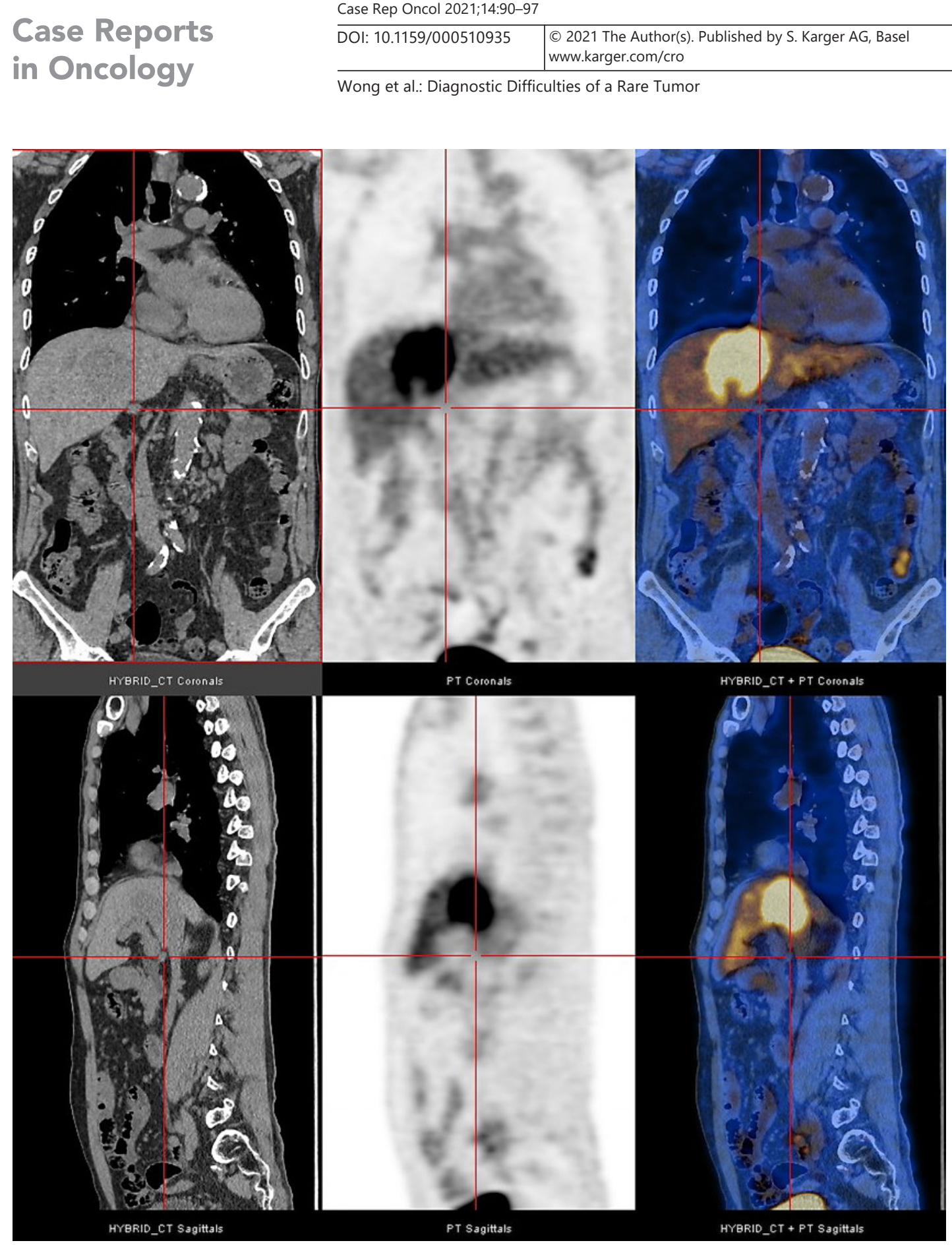

Fig. 2. ${ }^{18}$ FDG-PET scan showing a 7.4-cm hypermetabolic liver tumor with an SUVmax of 9.6. No other focal FDG-avid hypermetabolic lesion was detected.

Guidelines for the Diagnosis of Neuroendocrine Tumor, the tumor should be classified as an atypical carcinoid [4].

In view of the pathological findings, other investigations were performed after the operation to search for the possibility of remote primary NETs. The postoperative chromogranin level was elevated at $696 \mathrm{ng} / \mathrm{mL}$, and the 24 -h urine for 5 -hydroxyindoleacetic acid level was normal. Upper endoscopy with biopsy showed no malignancy. A dual-tracer PET scan with ${ }^{18} \mathrm{~F}$-FDG and ${ }^{68} \mathrm{Ga}$-DOTATATE showed mildly hypermetabolic lymph nodes in the right pericardial, subcarinal, para-aortic, right supraclavicular fossa, and bilateral axillary regions with 


\section{Case Reports in Oncology}

Fig. 3. Low-power view showing involvement of the liver parenchymal resection margin by the tumor.

Fig. 4. HE stain showing a well-circumscribed interface between the NET (lower field) and normal liver. Original magnification $\times 100$. The tumor cells form nests or tubules separated by fine fibrous bands. The cells have oval nuclei and eosinophilic cytoplasm.

Fig. 5. Higher-power view showing tumor cells with distinct nuclear and cytoplasmic membranes and prominent nucleoli. Original magnification $\times 400$.

\begin{tabular}{l|l}
\hline Case Rep Oncol 2021;14:90-97 \\
\hline DOI: 10.1159/000510935 & $\begin{array}{l}\text { @ 2021 The Author(s). Published by S. Karger AG, Basel } \\
\text { www.karger.com/cro }\end{array}$ \\
\hline
\end{tabular}
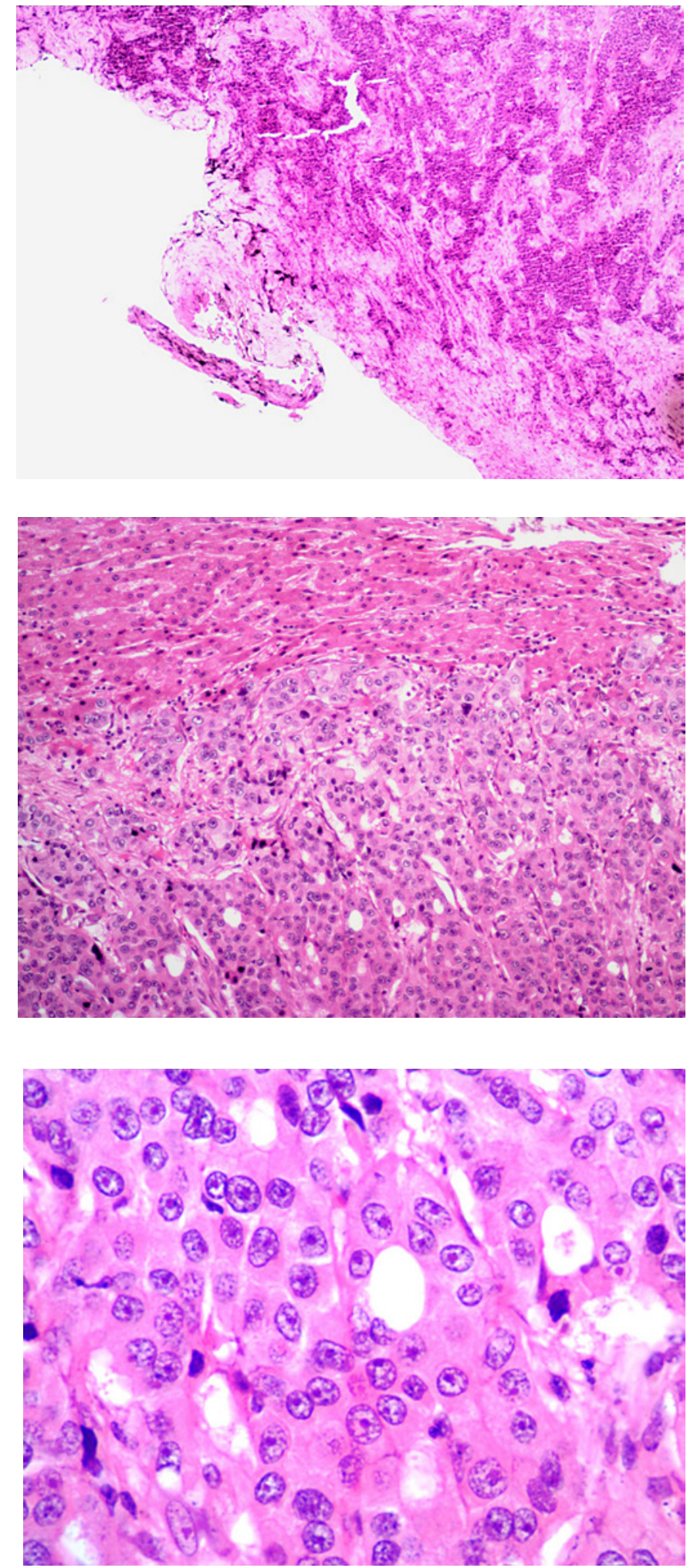


\section{Case Reports in Oncology}

\begin{tabular}{l|l}
\hline Case Rep Oncol 2021;14:90-97 \\
\hline DOI: 10.1159/000510935 & $\begin{array}{l}\text { ○ 2021 The Author(s). Published by S. Karger AG, Basel } \\
\text { www.karger.com/cro }\end{array}$ \\
\hline
\end{tabular}

Wong et al.: Diagnostic Difficulties of a Rare Tumor

Fig. 6. NET cells stain diffusely positive for chromogranin. Normal liver cells are negative.

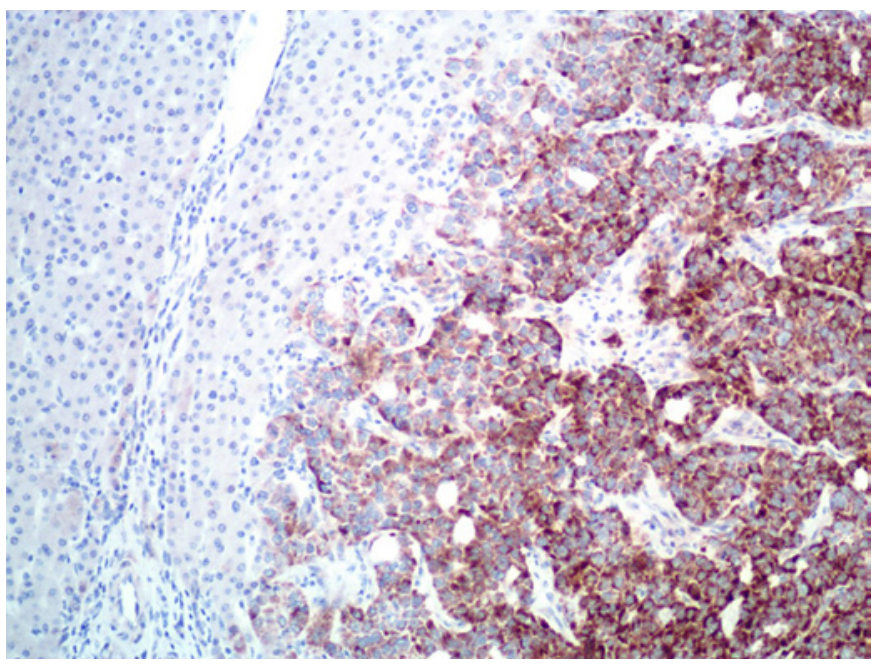

Fig. 7. HEPAR-1 stain highlights normal liver cells. NET cells are negative.

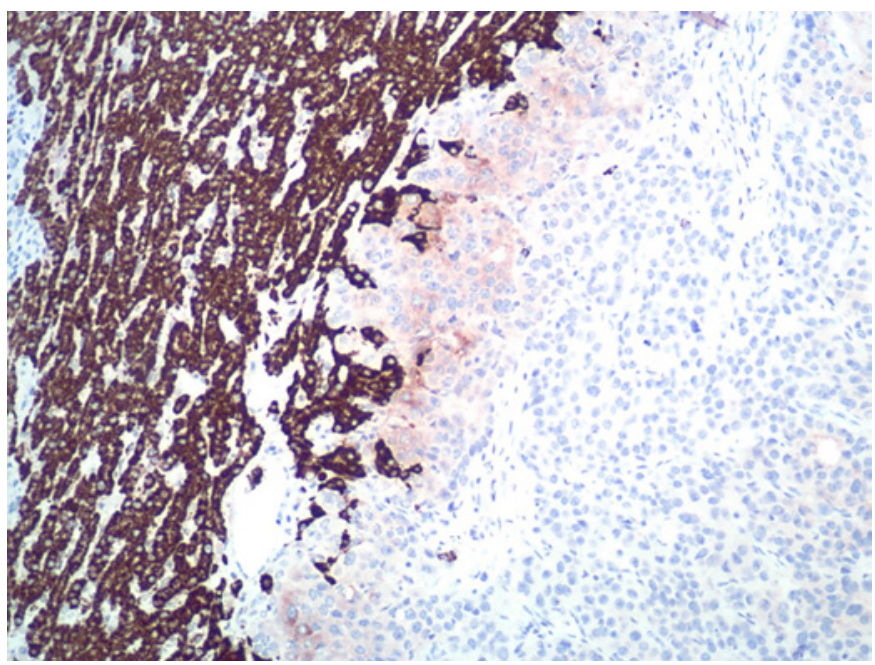

DOTATATE avidity and an SUVmax of $<4.2$. These findings were more in favor of a benign nature. There was no evidence of residual tumor in the liver remnant. Given the absence of other origins of NET, the final diagnosis of PHNET was confirmed. In view of the positive resection margin, the patient was given a monthly lanreotide injection. A reassessment CT scan was performed 8 months after the operation and confirmed no tumor recurrence. The patient survived for more than 16 months with a reasonably good quality of life.

\section{Discussion}

PHNET is an uncommon disease and evidence to guide its diagnosis and management was limited. A review of 124 patients with PHNET was performed by Quartey [1] in 2011. The mean age at diagnosis was 51.9 years with a slight female predominance. This demographic finding was echoed by another review in 2009 [5]. We report a case of PHNET in a patient with atypical demographics, including male gender, elderly, and a hepatitis B carrier. These clinical features would undoubtedly make HCC a probable diagnosis. It was demonstrated that the diagnosis of PHNET remained a challenging process and strategies for a better preoperative diagnosis should be investigated. 
Diagnosis of PHNET was often delayed as up to $26.7 \%$ of patients were asymptomatic [1]. Among symptomatic patients, 65 and $6.8 \%$ of patients presented with abdominal pain and classical carcinoid syndrome, respectively. The progression of disease is typically indolent and $12.5 \%$ of patients presented with an incidental finding of a large liver mass either during physical examination or imaging. A high index of suspicion for different causes of liver tumor should be maintained, especially in a patient with normal tumor markers despite a large liver tumor, regardless of the underlying hepatitis status.

Regarding characteristics of PHNET on CT scan, $74-88$ and $12-34 \%$ of tumors manifested as a hypodense mass or solid-cystic mass, respectively [5, 6]. In contrast to HCC, only $26 \%$ of them demonstrated arterial enhancement and portovenous washout, with peripheral enhancement being another feature for large tumor. The absence of this typical imaging characteristic for HCC would be further supporting evidence for other alternative diagnoses.

Other non-invasive tests should be followed for diagnosing PHNET, including serum chromogranin-A, 5-hydroxyindoleacetic acid (5-HIAA), and 24-h urine for 5-HIAA. The sensitivity and specificity of these biomarkers ranged from 73 to 80 and 95 to $100 \%$, respectively, according to the review by DeLuzio et al. [7].

In order to differentiate PHNET from other types of liver tumors, identification of somatostatin receptor overexpression using radiolabeled somatostatin analogue was crucial. Conventional somatostatin receptor scintigraphy (OctreoScan) using ${ }^{111}$ In-labeled DTPA-octreotide was shown to be effective in the diagnosis and staging of NETs, with a tumor detection rate between 80 and 100\% among studies [8]. With the advance in technology and increasing availability of PET scan, accuracy in the identification of NETs has been further enhanced. The serotonin precursor 11-C-5-hydroxytryptophan and somatostatin analogue ${ }^{68} \mathrm{Ga}$-DOTA peptides were the two commonly used PET tracers for NETs. PET scan was proven to achieve a better sensitivity ( $97 \%$ ) compared to $61 \%$ by CT and $52 \%$ by OctreoScan [9]. The utilization of FDG-PET in the detection of NETs was, however, limited by its intrinsically low metabolic rate and slow growth as compared with other carcinomas [10]. Nevertheless, FDG-PET should be performed for identification of metastases from other common liver tumors.

In addition to the diagnosis of tumor, PET scan also offered evaluation of tumor susceptibility to somatostatin analogue therapy through visualizing the overexpressed somatostatin receptors in tumor cells [9]. Its unique therapeutic implication played an important role in the subsequent management process.

Comparing PET against CT alone, Ruf et al. [11] suggested PET was superior to CT in terms of detection of lymph node, bone, and other organ metastases, whereas lung metastases and intestinal manifestations were more readily detectable on CT with intravenous contrast. Given the complementary nature of these two imaging modalities, the combination of CT and PET scan with ${ }^{18} \mathrm{~F}$-FDG and octreotide tracer should be recommended as a standard imaging protocol. The indication of surgery should be re-evaluated in case any primary NET or extrahepatic disease was identified preoperatively.

Liver biopsy was another controversial diagnostic tool. However, surgical resection remained the mainstay of treatment for localized PHNET, which achieved a 5-year survival and recurrence rate at 74 and 18\%, respectively [6]. Preoperative pathological diagnosis would not alter the operative planning while subjecting the patient to an additional risk of tumor seeding. Besides, the diagnostic accuracy was only $57.1 \%$ in the case series reported by Hwang et al. [12]. Needle biopsy should only be performed in the case of inoperability or presence of extrahepatic disease when liver metastasis has to be differentiated from a second primary malignancy. In these situations, liver biopsy would be valuable for the guidance of other oncological treatment.

Other than surgical resection, no well-defined treatment guideline was published due to scarcity of cases. For unresectable tumors, liver transplantation has been described in a few 
case reports with survival up to 95 months $[13,14]$. It was considered to be another possible curative treatment for patients with multifocal tumors and small liver reserves while better selection criteria are yet to be developed. Transarterial chemoembolization (TACE) was an alternative for unresectable tumors which offered palliation in terms of tumor load and symptom control; however, evidence was limited [15]. Huang et al. [16] reported the use of TACE in an adjuvant setting and tumor recurrence in 4 patients, which yielded an overall survival from 12 to 98 months.

The presence of somatostatin receptor in NET was a unique target facilitating the use of other systemic therapy. The efficacy of the long-acting somatostatin, lanreotide, on NETs has been well studied in terms of its anti-hormonal and anti-proliferative effect [17]. However, its use in treating PHNET was still uncertain due to the endocrinologically silent nature as compared with other gastrointestinal NETs presenting with carcinoid syndrome [12]. In this patient, lanreotide injection was shown to be effective in preventing disease recurrence after $\mathrm{R} 1$ resection of a large PHNET.

Peptide receptor radionuclide therapy (PRRT) with radiolabeled somatostatin analogue was another novel treatment targeting at somatostatin receptors in NET. Kwekkeboom et al. [18] reported 40-72 months of survival benefit compared with historical controls in patients with metastatic gastrointestinal NET treated with ${ }^{177}$ Lu-DOTATOC therapy. Another retrospective review demonstrated the effectiveness of ${ }^{177} \mathrm{Lu}$-DOTATE PRRT in achieving partial tumor response and stable disease particularly in a patient who has also received regional chemoembolization and radionuclide hepatic embolization [19]. The applicability of these promising results in metastatic NETs of hepatic origin should be investigated.

Systemic chemotherapy was mainly applied to metastatic disease. Despite no previous evidence of the efficacy of neoadjuvant treatment [20], the possibility of surgical resection following tumor downstaging by 5 -fluorouracil, etoposide, and cisplatin has been reported [21]. Randomized studies for comparison between each of these modalities in terms of treatment outcome and safety profile would be beneficial.

\section{Conclusion}

Accurate preoperative diagnosis of PHNET remained an important yet difficult feature in the management of this rare tumor. Dual-tracer PET-CT scan, blood, and urine tests for biomarkers are highly recommended for patients with suspected PHNET. Further studies regarding the efficacies of the diagnostic tests and various emerging treatment modalities are warranted.

\section{Statement of Ethics}

The authors have no ethical conflicts to disclose. Written informed consent was obtained from the patient for publication of this case report and accompanying images. The research was conducted ethically in accordance with the World Medical Association Declaration of Helsinki.

\section{Conflict of Interest Statement}

The authors declare no conflicts of interest.

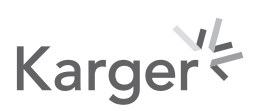




\section{Case Reports in Oncology}

\begin{tabular}{l|l}
\hline Case Rep Oncol 2021;14:90-97 \\
\hline DOI: 10.1159/000510935 & $\begin{array}{l}\text { ○ 2021 The Author(s). Published by S. Karger AG, Basel } \\
\text { www.karger.com/cro }\end{array}$ \\
\hline
\end{tabular}

Wong et al.: Diagnostic Difficulties of a Rare Tumor

\section{Funding Sources}

The authors have no funding sources to report.

\section{Author Contributions}

P.C.W.: acquisition of clinical data and interpretation, drafting of the manuscript. T.T.C. and W.H.S.: critical revision of the manuscript for intellectual content. U.S.K.: slide review and interpretation of pathology specimens.

\section{References}

1 Quartey B. Primary hepatic neuroendocrine tumor: what do we know now?. World J Oncol. 2011;2(5):209-16.

2 Urata K, Kawasaki S, Matsunami H, Hashikura Y, Ikegami T, Ishizone S, et al. Calculation of child and adult standard liver volume for liver transplantation. Hepatology. 1995;21(5):1317-21.

3 Chan SC, Liu CL, Lo CM, Lam BK, Lee EW, Wong Y, et al. Estimating liver weight of adults by body weight and gender. World J Gastroenterol. 2006;12(14):2217-22.

4 Kunz PL, Reidy-Lagunes D, Anthony LB, Bertino EM, Brendtro K, Chan JA, et al. Consensus Guidelines for the Management and Treatment of Neuroendocrine Tumors, North American Neuroendocrine Tumor Society. Pancreas. 2013;42(4):557-77.

5 Lin CW, Lai CH, Hsu CC, Hsu CT, Hsieh PM, Hung KC, et al. Primary hepatic carcinoid tumor: a case report and review of the literature. Cases J. 2009;2(1):90.

6 Iwao M, Nakamuta M, Enjoji M, Kubo H, Fukutomi T, Tanabe Y, et al. Primary hepatic carcinoid tumor: case report and review of 53 cases. Med Sci Monit. 2001;7(4):746-50.

7 DeLuzio MR, Barbieri AL, Israel G, Emre S. Two cases of primary hepatic neuroendocrine tumors and a review of the current literature. Ann Hepatol. 2017;16(4):621-9.

8 Oberg K, Eriksson B. Nuclear medicine in the detection, staging and treatment of gastrointestinal carcinoid tumours. Best Pract Res Clin Endocrinol Metab. 2005;19(2):265-76.

9 Ambrosini V, Campana D, Tomassetti P, Fanti S. 68Ga-labelled peptides for diagnosis of gastroenteropancreatic NET. Eur J Nucl Med Mol Imaging. 2012;39(Suppl 1):S52-60.

10 Gabriel M, Decristoforo C, Kendler D, Dobrozemsky G, Heute D, Uprimny C, et al. 68Ga-DOTA-Tyr3-octreotide PET in neuroendocrine tumors: comparison with somatostatin receptor scintigraphy and CT. J Nucl Med. 2007;48(4):508-18.

11 Ruf J, Heuck F, Schiefer J, Denecke T, Elgeti F, Pascher A, et al. Impact of multiphase 68Ga-DOTATOC-PET/CT on therapy management in patients with neuroendocrine tumors. Neuroendocrinology. 2010;91(1):101-9.

12 Hwang S, Lee YJ, Lee SG, Kim CW, Kim KH, Ahn CS, et al. Surgical treatment of primary neuroendocrine tumors of the liver. J Gastrointest Surg. 2008;12(4):725-30.

13 Gurung A, Yoshida EM, Scudamore CH, Hashim A, Erb SR, Webber DL. Primary hepatic neuroendocrine tumour requiring live donor liver transplantation: case report and concise review. Ann Hepatol. 2012;11(5):715-20.

14 Fenwick SW, Wyatt JI, Toogood GJ, Lodge JP. Hepatic resection and transplantation for primary carcinoid tumors of the liver. Ann Surg. 2004;239(2):210-9.

15 Touloumis Z, Delis SG, Triantopoulou C, Giannakou N, Avgerinos C, Dervenis C. Primary hepatic carcinoid; a diagnostic dilemma: a case report. Cases J. 2008;1(1):314.

16 Huang YQ, Xu F, Yang JM, Huang B. Primary hepatic neuroendocrine carcinoma: clinical analysis of 11 cases. Hepatobiliary Pancreat Dis Int. 2010;9(1):44-8.

17 Wängberg B, Nilsson O, Johanson V, Kölby L, Forssell-Aronsson E, Andersson P, et al. Somatostatin receptors in the diagnosis and therapy of neuroendocrine tumor. Oncologist. 1997;2(1):50-8.

18 Kwekkeboom DJ, de Herder WW, Kam BL, van Eijck CH, van Essen M, Kooij PP, et al. Treatment with the radiolabeled somatostatin analog [177Lu-DOTA0,Tyr3]octreotate: toxicity, efficacy, and survival. J Clin Oncol. 2008;26(13):2124-30.

19 Hamiditabar M, Ali M, Bolek L, Vahdati G, Tworowska I, Delpassand ES. Safety and effectiveness of 177Lu-DOTATATE peptide receptor radionuclide therapy after regional hepatic embolization in patients with somatostatin-expressing neuroendocrine tumors. Clin Nucl Med. 2017;42(11):822-8.

20 Knox CD, Anderson CD, Lamps LW, Adkins RB, Pinson CW. Long-term survival after resection for primary hepatic carcinoid tumor. Ann Surg Oncol. 2003;10(10):1171-5.

21 Park CH, Chung JW, Jang SJ, Chung MJ, Bang S, Park SW, et al. Clinical features and outcomes of primary hepatic neuroendocrine carcinomas. J Gastroenterol Hepatol. 2012;27(8):1306-11. 